\section{Neuromyelitis optic with positive Anti-AQP4 and Anti-SSA/Ro antibody}

Riwanti Estiasari, ${ }^{1}$ Salsabila Firdausia,1 Dewi Wulandari, ${ }^{2}$ Kartika Maharani, ${ }^{1}$ Freddy Sitorus, ${ }^{1}$ David Pangeran, ${ }^{1}$ Darma Imran 1

1Department of Neurology, and 2Department of Clinical Pathology, Faculty of Medicine, Universitas Indonesia, dr. Cipto Mangunkusumo General Hospital, Jakarta, Indonesia

\section{Abstract}

Neuromyelitis Optic (NMO) is an inflammatory disorder involving central nervous system which often co-exists with other autoimmune diseases such as Sjögren's syndrome (SS). NMO manifestation could precede or follow SS, but the role of anti-SSA in the pathogenesis of NMO remains unclear. We present a case of NMO with anti-AQP4 anti-SSA antibody positive. A-44-year-old female presented with right side weakness. The symptoms began with numbness that improved spontaneously. She also complained pain and dry sensations on her eyes. Schirmer test on her left eye, antinuclear antibody (ANA) and antiSSA antibody were positive. Cervical MRI revealed intramedullary lesion on T2weighted-image at C2-C5 level. She was diagnosed as NMO with positive antiAQP4 and probable SS. She received $1 \mathrm{~g}$ methylprednisolone for 5 days proceeded with mycophenolic acid. One-year observation showed clinical improvement. Systemic autoantibodies must substantially be evaluated in NMO. Comprehensive diagnosis and providing appropriate immunosuppressant might prevent further disability and relapse.

\section{Introduction}

Neuromyelitis optica (NMO) is an inflammatory disorder of the central nervous system (CNS) that mostly involve optic nerve and spinal cord. Prior to the discovery of anti-aquaporine 4 (AQP4) antibodies, NMO was considered as a variant of multiple sclerosis (MS) due to their similar symptoms. However, it has already been recognized that both diseases have different pathophysiology and management. ${ }^{1}$ Unlike MS, the presence of NMO is often associated with other autoimmune diseases such as systemic lupus erythematosus (SLE) and Sjögren's syndrome (SS). ${ }^{2}$

Neurologic manifestations, including peripheral neuropathy, optic neuritis, and transverse myelitis, are found in approximately $20 \%$ of SS cases. ${ }^{3}$ Several studies also showed positive anti-AQP4 antibody in some cases of SS.4,5 However, it is still debated whether NMO is a neurological complication of SS or coexists with SS. ${ }^{6}$

Here, we report a 44-year-old female with NMO who had positive anti-AQP4 and anti-SSA antibody which responded well with immunosuppressant. Although NMO is still considered as a rare disease in Indonesia, the number of cases keeps increasing. Early recognition, comprehensive diagnosis, and appropriate treatment might prevent further disability.

\section{Case Report}

A 44-year old female, mixed race between Tapanuli and Chinese, came to our hospital with weakness on her right arm and leg since a month ago. The symptoms were preceded by fluctuating tingling and numbness on her right leg that sometimes resolved spontaneously. There was headache, which she described as being electrified. She also experienced dry eye and pain with gray cloud-like shadow on her left eye which worsen in the morning and after heat exposure. No sand sensation was reported on her left eyes. Fever or other diseases were denied, but fatigue was perceived almost every day.

Twenty years ago, she also had similar symptoms of weakness on her right side and difficulty to move her eyeball to the left which resolved spontaneously.

Neurologic examinations revealed weakness and hypesthesia on her right leg accompanied with increased physiological reflexes and bilateral positive Hoffmann and Tromnner signs. Her visual acuity was within the normal limit on her right eye but decreased on the left one (1/60). There were relative afferent pupillary defect and temporal pallor on the left optic nerve head that indicated optic nerve atrophy. Other cranial nerves were within normal limit. Schirmer test showed $15 \mathrm{~cm}$ moisture on the right eye but only $5 \mathrm{~cm}$ on the left eye in 5 minutes.

Cervical MRI revealed T2-weighted hyperintensity on segment C3-C5 without gadolinium enhancement (Figure 1). Brain MRI result was normal.

Cerebrospinal fluid (CSF) analysis was also within normal limit. Herpes simplex, varicella zoster, Epstein-Barr and Mycobacterium tuberculosis DNA were not detected on polymerase chain reaction
Correspondence: Riwanti Estiasari, Department of Neurology Faculty of Medicine Universitas Indonesia, Jl. Salemba Raya No. 4, Central Jakarta 14310, Indonesia.

Tel.: +62.81806006061

E-mail: riwanti.estiasari04@ui.ac.id

Key words: Neuromyelitis Optic, Sjogren, AQP4, Anti-SSA.

Contributions: the authors contributed equally.

Conflict of interest: the authors declare no potential conflict of interest.

Funding: This report is supported by Hibah Pitta 2018 (PITTA/694/FK/2018) funded by DRPM Universitas Indonesia.

Received for publication: 29 November 2018 Accepted for publication: 9 December 2018.

This work is licensed under a Creative Commons Attribution NonCommercial 4.0 License (CC BY-NC 4.0).

(C) Copyright R. Estiasari et al., 2019

Licensee PAGEPress, Italy

Neurology International 2019; 11:7958

doi:10.4081/ni.2019.7958

(PCR). The oligoclonal band was negative both in CSF and serum (Table 1).

Indirect immunofluorescence antinuclear antibody (IF-ANA) test showed speckled pattern with the titer of 1:320. Serum anti-Ro/SSA antibody was strongly positive with borderline anti-La/SSB and negative anti ds-DNA antibody.

Anti-AQP4 antibody was examined in the Clinical Pathology Laboratory of Cipto Mangunkusumo Hospital using cell-based indirect immunofluorescence assay utilizing M1-AQP4-transfected HEK293 cells. The result was positive as seen in Figure 2.

The patient was diagnosed as NMOSD with positive anti-AQP4 antibody and probable SS. Following the administration of $1,000 \mathrm{mg}$ methylprednisolone for five days, her clinical condition improved. The treatment was continued with mycophenolic acid $360 \mathrm{mg}$ bid. No relapse occurred in a year of observation.

\section{Discussion}

NMO, previously known as Devic's disease, is an autoimmune disease characterized by optic neuritis and longitudinally extensive transverse myelitis ( $\geq 3$ vertebral segments) (LETM). Its prevalence in Southeast Asia is estimated to be 0.43 $2.7 / 100,000$ population. ${ }^{1}$ 
The pathogenesis of NMO is mediated by anti-AQP4 antibody which caused astrocyte damage. AQP4 is a type-III transmembrane protein that regulates water transportation in the brain cells. It was abundant in the astrocyte membrane of bloodendothelial barrier and blood-CSF barrier. It is commonly found in the CNS and is mostly expressed in retina, optic nerve, hypothalamus, cerebellum, periventricular, periaqueductal region, and spinal cord.2,3

Anti-AQP4 antibody, known as NMO immunoglobulin (NMO-IgG), is an isotype of IgG1 which had potencies to activate complement system and produced C5b-9 lytic complex resulting in the destruction of the astrocyte. 2,4 The active lesion of NMO showed loss of AQP4 molecules and perivascular glial fibrillary acid proteins

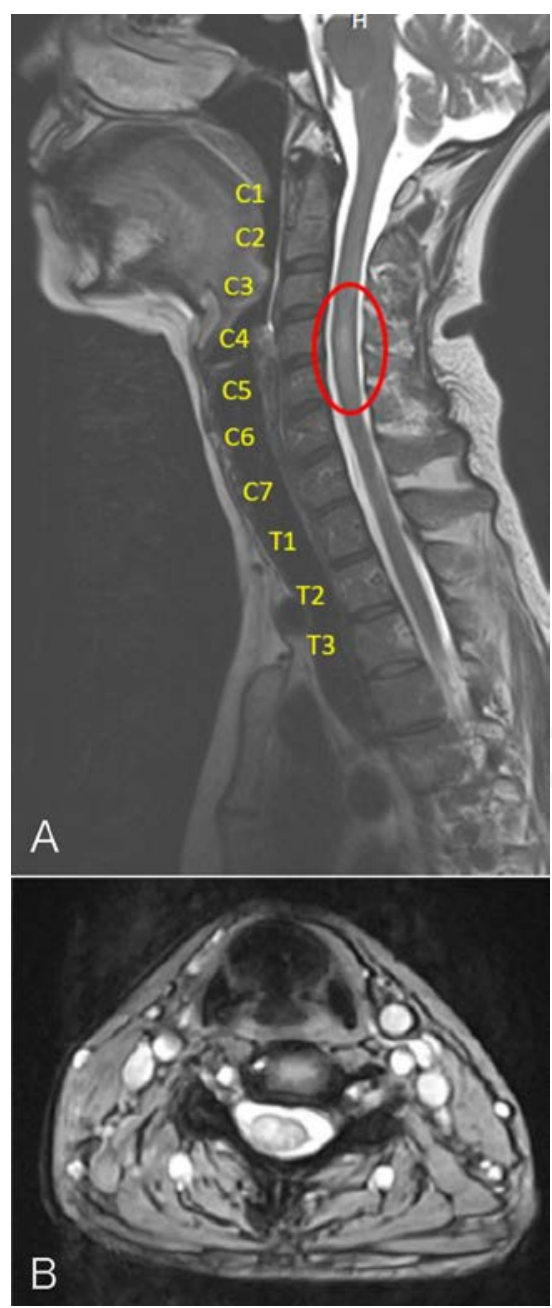

Figure 1. Cervical magnetic resonance imaging. A) The sagittal T2-weighted image showed hyperintensity in the spinal cord at level C3-C5. B) The axial T2 weighted image on the level of $\mathrm{C} 4$ showed hyperintensity in the center of the spinal cord.
(GFAP). ${ }^{2}$ GFAP is an intermediate filament which plays an important role for cytoskeletal and mechanic strength of the astrocyte. It also supports the function of adjacent neuron and the blood-brain barrier. 5

Unlike MS, NMO is more prevalent in the non-caucasian population. The predisposing genetic factors in NMO also differed from MS. In Brazilian, anti-AQP4 antibody seropositivity is associated with human leukocyte antigen (HLA)-DRB1*03 (DR3) 6 whereas in Japanese and Chinese, it is associated with HLA-DPB $1 * 0501.7,8$

The International Panel for NMO Diagnosis (IPND) has developed revised NMO diagnostic criteria which includes the anti-AQP4 antibody status. ${ }^{9}$ According to these criteria, this patient fulfills the criteria for NMOSD with positive anti-AQP4. She has two major criteria, optic neuritis, and transverse myelitis. The anti-AQP4 antibody was also positive, tested using indirect immunofluorescence cell-based assay.

NMO often co-exists with other systemic autoimmune diseases including SLE and SS. 10 Park et al. reported that of 48 NMO patients with positive anti-AQP4 antibody, 18 patients $(37.5 \%)$ had concomitant positive anti-SS-A antibody. The seropositivity of anti-AQP4 antibody was also higher in anti-SSA seropositive subjects compared with the seronegative ones. ${ }^{11}$ Estiasari et al. also found that antiAQP4 antibody was detected in $31.8 \% \mathrm{SS}$ patients, of which $85.7 \%$ had LETM lesion. ${ }^{12}$ Similar result was also reported from Korea, in which of 106 anti AQP4 patients, 33\% had other autoantibodies such as ANA and anti-SSA. ${ }^{13}$ The role of antiSSA in the pathogenesis of NMO is still a focus of debate. Anti-AQP4 antibody is expressed not only in the CNS, but also in the kidney, gastrointestinal, and respiratory tract, but we found no report on extra-CNS organ involvement in NMO patients. It seems that the autoantibody pathogenicity is only restricted in the CNS milieu and it has already known that anti-AQP4 antibody can destruct astrocyte. ${ }^{2}$ However, antiAQP4 antibody can be detected in the serum long before the clinical manifestation of NMO appears. Nishiyama et al reported a case of 34-year-old female whose antiAQP4 antibody has been already detected ten years before the NMO. ${ }^{14}$ Anti-AQP4 antibody also persists in the remission phase of NMO. 15

According to these two pieces of evidence, we concluded that anti-AQP4 antibody can only cause the disease if it penetrates the blood-brain barrier (BBB). Under physiologic condition, anti-APQ4 antibody did not cross the BBB. It has been hypothesized that other triggers or pre-existing CNS inflammation are essential to break the BBB that allow the anti-AQP4 antibody to enter the brain. ${ }^{2-4}$ SSA antigen is expressed in the endothelial cells. The reaction between SSA antigen with the antibody will lead to disruption of the endothelial cells

Table 1. Cerebrospinal fluid analysis result.

\begin{tabular}{|c|c|c|}
\hline CSF analysis & Result & Reference \\
\hline 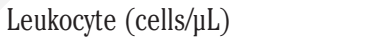 & 6 & $0-5$ \\
\hline Polymorphonuclear (cells/ $\mu \mathrm{L}$ ) & 5 & - \\
\hline 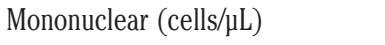 & 95 & - \\
\hline Glucose (mg/dL) & 78 & $45-80$ \\
\hline Protein (mg/dL) & 27.5 & $15-45$ \\
\hline Herpes Simplex type I and II DNA & Not detected & Not detected \\
\hline Cytomegalovirus DNA & Not detected & Not detected \\
\hline Epstein-Barr virus DNA & Not detected & Not detected \\
\hline Mycobacterium Tuberculosis DNA & Not detected & Not detected \\
\hline
\end{tabular}

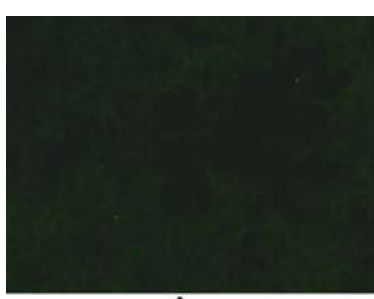

A

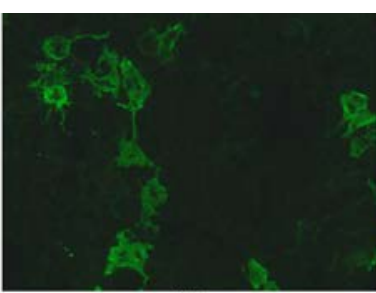

B

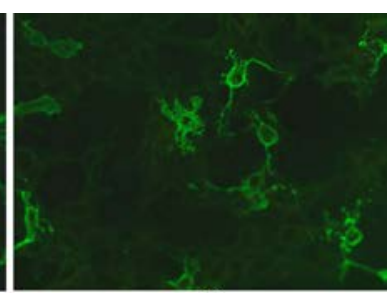

C

Figure 2. Cell-based indirect immunofluorescence assay for anti-AQP4 antibody detection. A) negative control; B) positive control; C) Patient serum. 
including brain endothelial cells. It might cause the leakage of BBB which provides anti-AQP4 antibody to enter the brain parenchyma. ${ }^{11}$ Despite the detection of antiSSA antibody in our case, the criteria to definitely diagnose SS were not fully fulfilled. She experienced dry eyes for two months with positive Schirmer's test on the left eye, but no dry mouth and histopathologic evidence in this case. NMO symptoms can precede the diagnosis of SS as reported by Park et al. which discovered that of 21 patient who fulfills the diagnostic criteria for SS, five were diagnosed in the initial evaluation of NMO and 18 were in the follow-up phase.11 Our patient showed clinical improvement and no relapse in one-year observation with immunosuppressant. She was initially treated with high-dose methylprednisolone, which was followed with mycophenolic acid. Commonly-used immunosuppressant for NMO includes azathioprine, mycophenolic acid, and rituximab. ${ }^{16}$ A prospective cohort study comparing the efficacy of azathioprine, mycophenolate mofetil, and low-dose rituximab discovered the similarity on the effectiveness among these medications in reducing ARR (Annualized Relapse Rate) and improving the clinical symptoms. Mycophenolate mofetil and rituximab also decrease the anti-AQP4 antibody titer and provide less side effects compared with azathioprine. ${ }^{17}$

\section{Conclusions}

Co-existence of NMO and SS is common with the positivity of both anti AQP4 and anti-SSA antibody, hence evaluation of systemic autoantibody in NMO cases is important. The mechanism of the role of anti-SSA antibody in the pathogenesis of NMO remains to be elucidated. Appropriate immunosuppressant treatment is beneficial in achieving disease remission and minimizes the disability.

\section{References}

1. Pandit L, Asgari N, Apiwattanakul M, et al. Demographic and clinical features of neuromyelitis optica: A review. Mult Scler J 2015;21:845-53.

2. Bukhari W, Barnett MH, Prain K, Broadley SA. Molecular Pathogenesis of Neuromyelitis Optica. Int J Mol Sci 2012;13:12970-93.

3. Nagelhus EA, Ottersen OP. Physiological Roles of Aquaporin-4 in Brain. Physiol Rev 2013;93:1543-62.

4. Kinoshita M, Nakatsuji Y. Where Do AQP4 Antibodies Fit in the Pathogenesis of NMO? [Internet]. Multiple Sclerosis International. 2012 [cited 2018 Nov 3]. Available from: https://www.hindawi.com/journals/msi/ 2012/862169/

5. McKeon A, Benarroch EE. Glial fibrillary acid protein: Functions and involvement in disease. Neurology 2018;90:925-30.

6. Guimaraães Brum D, Barreira AA, et al. HLA-DRB association in neuromyelitis optica is different from that observed in multiple sclerosis. Mult Scler J 2010;16:21-9.

7. Matsushita $T$, Matsuoka $T$, Isobe $N$, et al. Association of the HLA-DPB $1 * 0501$ allele with anti-aquaporin-4 antibody positivity in Japanese patients with idiopathic central nervous system demyelinating disorders. Tissue Antigens 2009;73:171-6.

8. Wang H, Dai Y, Qiu W, et al. HLADPB $1 * 0501$ is associated with susceptibility to anti-aquaporin-4 antibodies positive neuromyelitis optica in Southern Han Chinese. J Neuroimmunol 2011;233:181-4.

9. Wingerchuk DM, Banwell B, Bennett $\mathrm{JL}$, et al. International consensus diagnostic criteria for neuromyelitis optica spectrum disorders. Neurology 2015;85:177-89.
10. Jarius S, Jacobi $C$, de Seze J, et al. Frequency and syndrome specificity of antibodies to aquaporin-4 in neurological patients with rheumatic disorders. Mult Scler J 2011;17:1067-73.

11. Park JH, Hwang J, Min JH, et al. Presence of anti-Ro/SSA antibody may be associated with anti-aquaporin-4 antibody positivity in neuromyelitis optica spectrum disorder. J Neurol Sci 2015;348:132-5.

12. Estiasari R, Matsushita T, Masaki K, et al. Comparison of clinical, immunological and neuroimaging features between anti-aquaporin-4 antibody-positive and antibody-negative Sjögren's syndrome patients with central nervous system manifestations. Mult Scler J 2012;18:807-16.

13. Kim SH, Kim W, Li XF, et al. Clinical spectrum of CNV aquaporin-4 autoimmunity. Neurology 2012;78:1179-85.

14. Nishiyama S, Ito T, Misu T, et al. A case of NMO seropositive for aquaporin-4 antibody more than 10 years before onset. Neurology 2009;72:1960-1.

15. Matsushita $T$, Isobe $\mathrm{N}$, Matsuoka $\mathrm{T}$, et al. Aquaporin-4 autoimmune syndrome and anti-aquaporin-4 antibody-negative opticospinal multiple sclerosis in Japanese. Mult Scler J 2009;15:834-47.

16. Kessler RA, Mealy MA, Levy M. Treatment of Neuromyelitis Optica Spectrum Disorder: Acute, Preventive, and Symptomatic. Curr Treat Options Neurol 2016;18:2.

17. Yang Y, Wang C, Wang B, et al. Comparison of efficacy and tolerability of azathioprine, mycophenolate mofetil, and lower dosages of rituximab among patients with neuromyelitis optica spectrum disorder. J Neurol Sci 2018;385: 192-7. 